\title{
Interventive radiology in portal hypertension
}

\section{S Beningfield, MB ChB, FFRad (D)}

Department of Radiology, University of Cape Town

\section{Introduction}

Portal hypertension can be successfully managed using a variety of interventional radiology techniques. The presinusoidal, sinusdoidal and post sinusoidal categories have different options available, as does segmental portal hypertension. The major role of TIPS has been tempered by early occlusions and high rates of hepatic encephalopathy.

\section{Imaging}

The standard subdivisions of portal hypertension into pre-sinusoidal, sinusoidal and postsinusoidal groups may be well evaluated using Duplex Doppler and multislice CT. MRI and angiography are reserved for more complicated cases, especially those requiring intervention.

A useful adjunct in planning therapy is the retrograde portogram via wedged hepatic vein catheter. In particular where there is portal vein thrombosis/ cavernous transformation, the flow and diameter of the portal vein are reduced, allowing a better quality study (personal communication - Jean de Ville de Goyet). Carbon dioxide retrograde portography has been used in planning TIPS placement, as the lower viscosity of carbon dioxide favours easier retrograde portal vein filling.

\section{Intervention}

The options for intervention involve reducing inflow, eliminating gradients within the system, isolating vessels that are sources of haemorrhage and increasing outflow.

\section{Segmental}

This includes opening up thrombosed or stenosed splenic, superior mesenteric or main portal veins. For acute mesenteric and portal thrombosis, successful clearance by mechanical and chemical thrombolysis has been reported. ${ }^{1}$ Thrombolytic agents can be delivered either by arterial or venous routes; the former may lead to wider

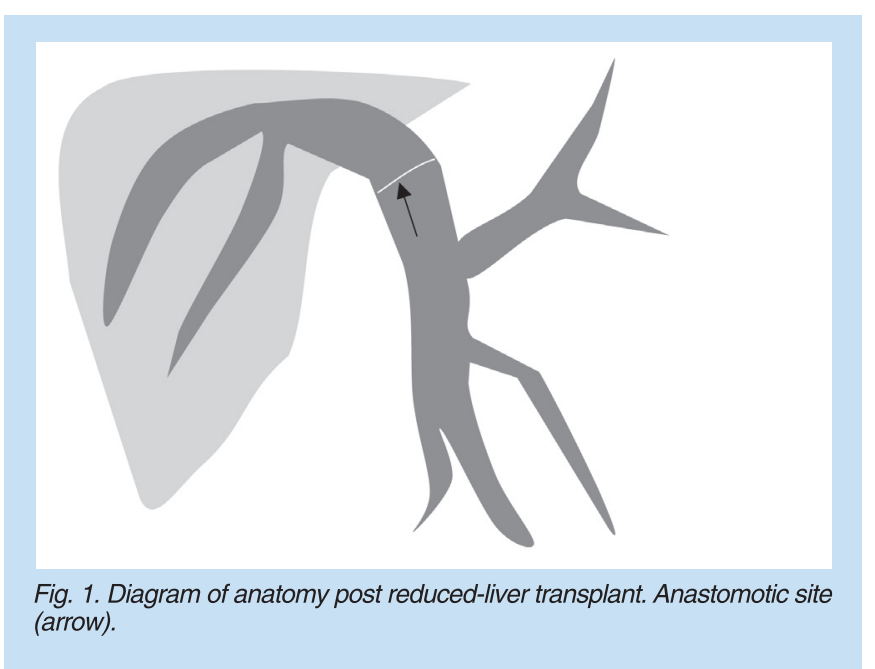

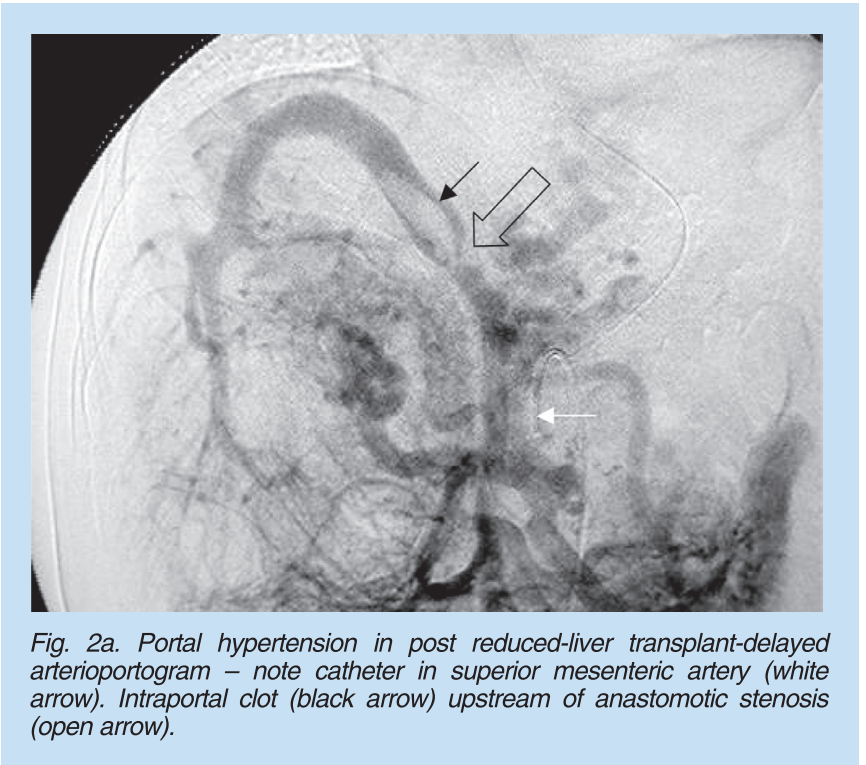
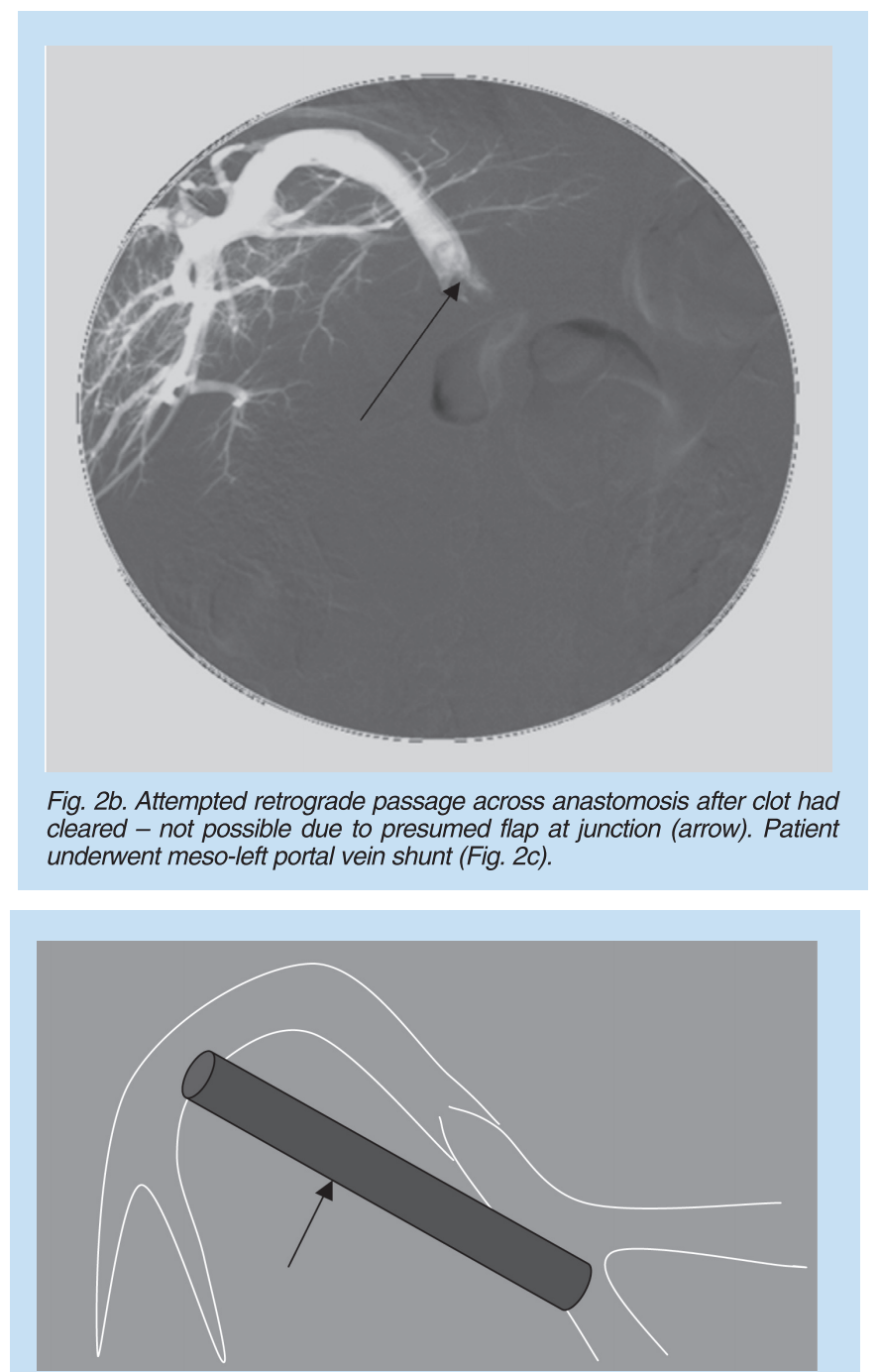

Fig. 2c. Patient underwent meso-left portal vein shunt (arrow). 


\section{PICTORIAL REVIEW}
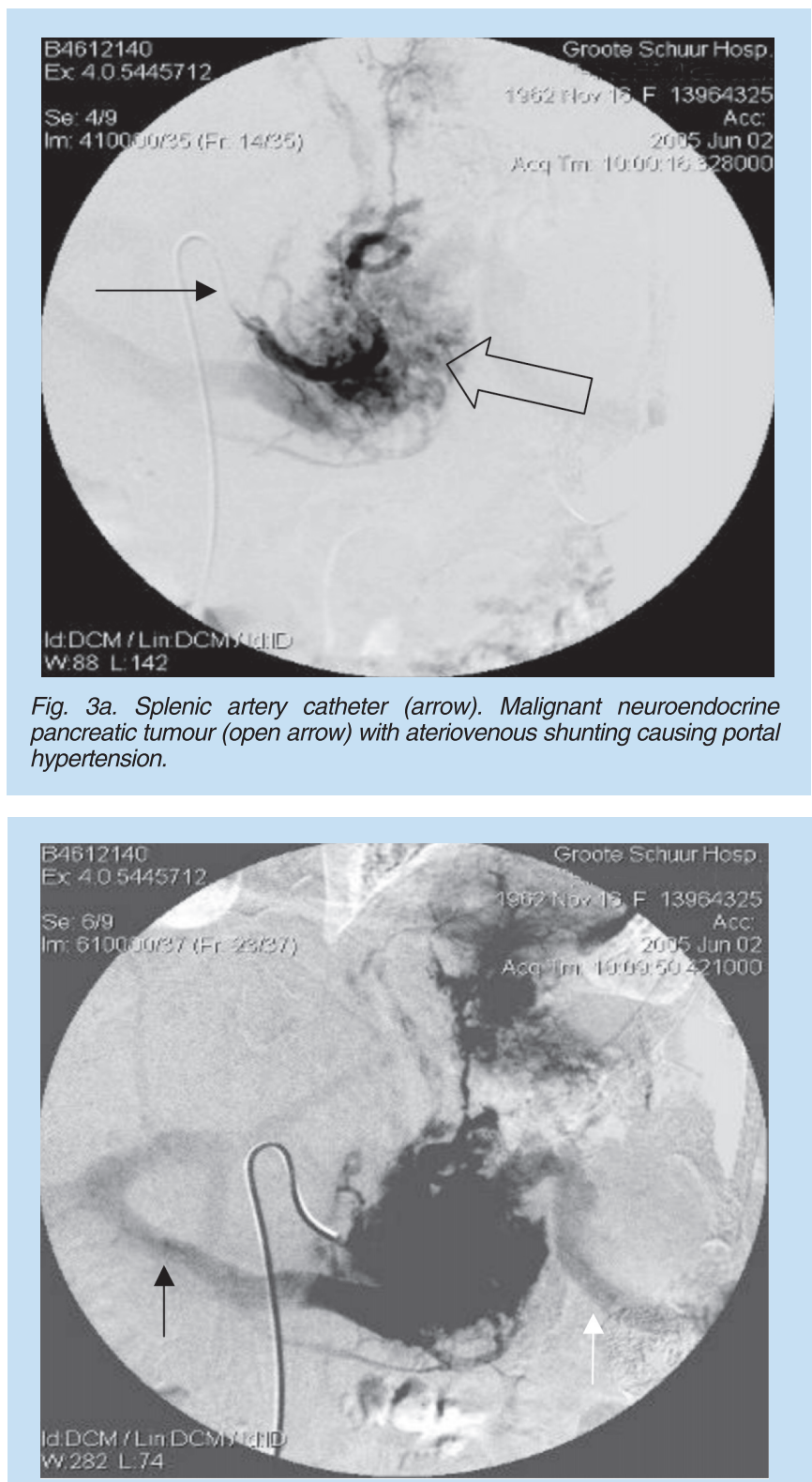

Fig. 3b. Later phase showing early opacification of splenic (white arrow) and portal vein (black arrow)

action, but with the risk of bypassing occluded areas; the venous route produces higher concentrations but in a limited region. However, before embarking on lysis, it is important to consider the risks of aggravated or induced haemorrhage in the area of the gut drained by the affected veins.

Stenoses, such as those related to chronic pancreatitis, may respond to angioplasty and stenting via transhepatic or transjugular routes. The other major category of stenotic segmental portal hypertension is in the paediatric post-transplant setting, usually due to a stenosis at the portal vein anastomosis site between donor and recipient portal veins (Figs $1,2)$. The outcome of interventional venoplasty in this category was successful in $71 \%$ in one series, and appears reasonable in the medium term. $^{2}$

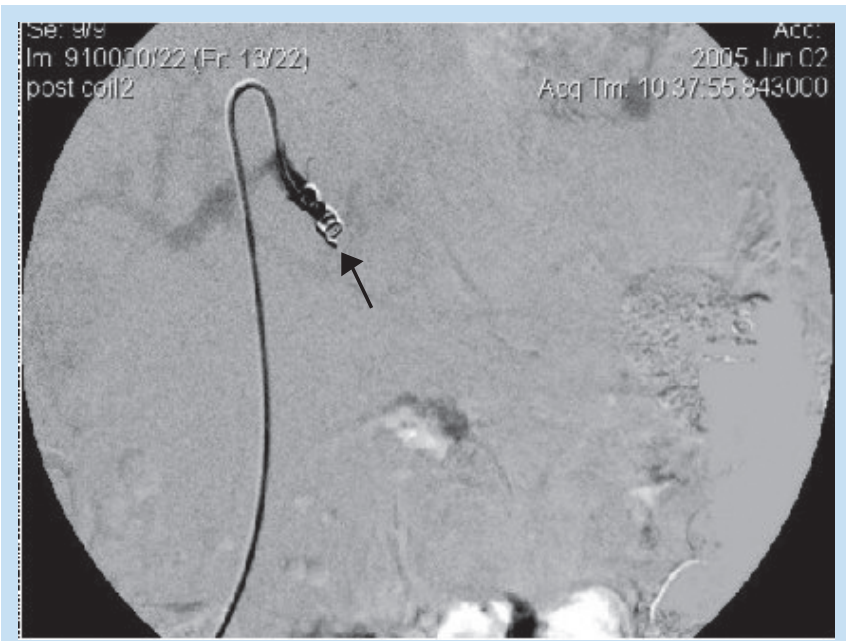

Fig. 3c. Post embolisation of splenic artery with coils (arrow). No shunting seen.

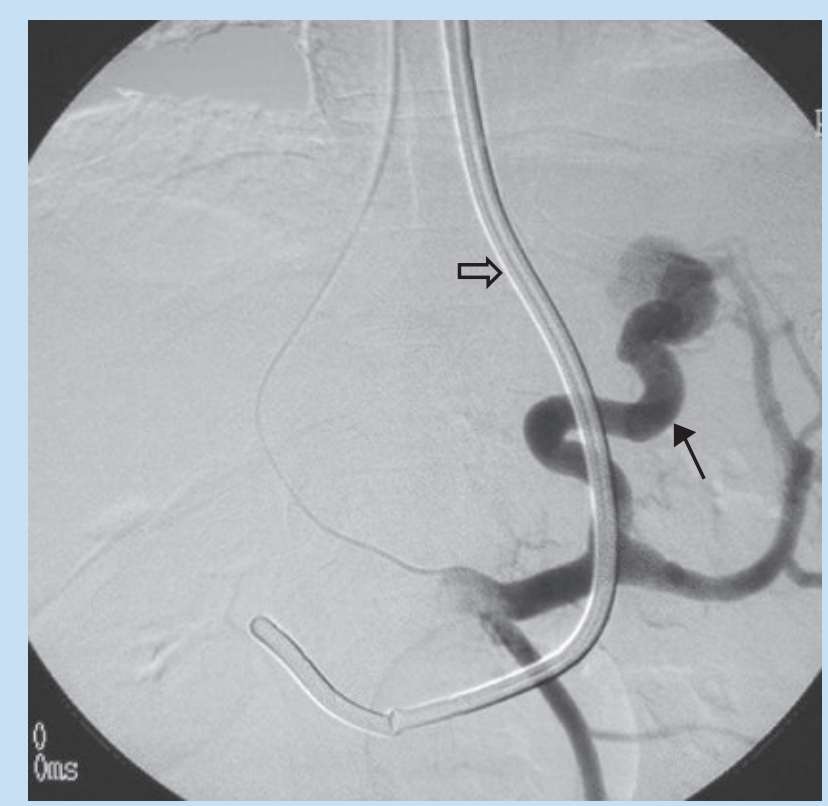

Fig. 4a. Transjugular portogram during TIPS with filling of left gastric vein (arrow). Note Sengstaken tube (open arrow).

\section{Presinusoidal}

The rare splenic arteriovenous malformation is a logical lesion to embolise and occlude, to reduce the resultant arteriovenous shunting and portal hypertension.

Embolisation of a large but otherwise normal spleen can reduce the hyperdynamic effects of hypersplenism, including reducing inflow into the portal system. Reduced platelet consumption is a further benefit. This can be achieved, although with the risk of complications in some series, ${ }^{3}$ particularly sepsis and even mortality.

Arterioportal shunts, whether post-traumatic or following biopsy, are also amenable to embolisation, ideally using a controllable embolisation system such as a detachable coil. Hypervascular tumours, 


\section{PICTORIAL REVIEW}

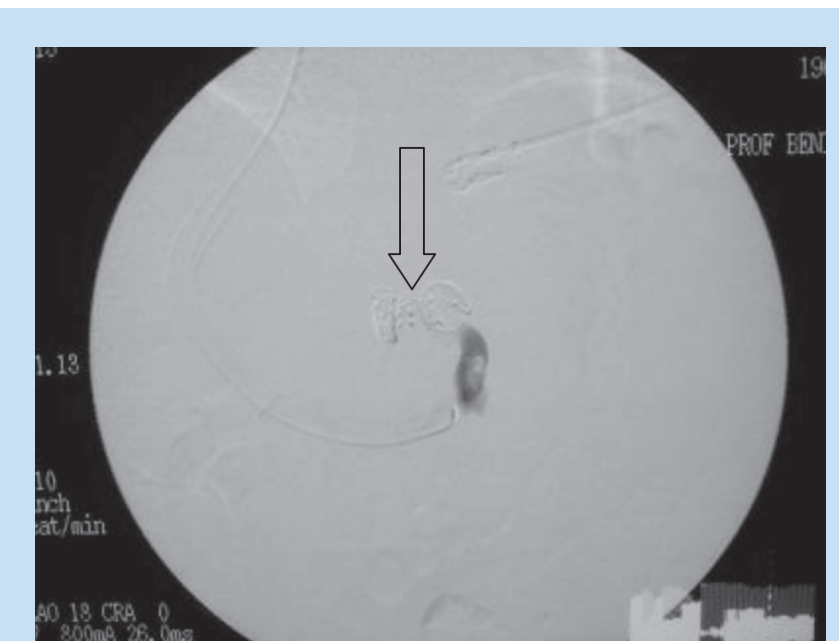

Fig. 4b. Occlusion of left gastric vein with coils (open arrow).

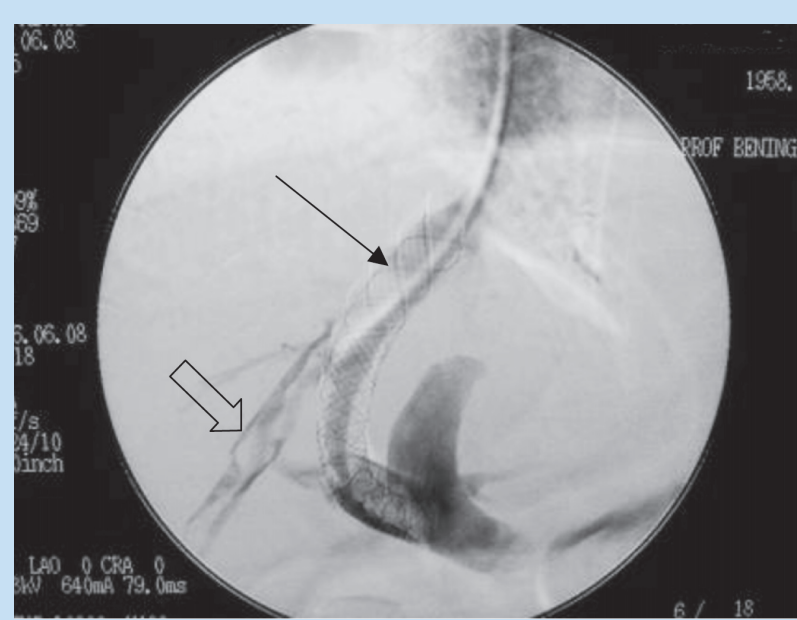

Fig. 5a. Partially thrombosed TIPS shunt (arrow) and right hepatic vein (open arrow).

such as malignant neuroendocrine pancreatic tumours, can also rarely lead to portal hypertension by arteriovenous shunting. Pre-operative embolisation can reduce the local portal hypertension, facilitating resection (Fig. 3).

\section{Sinusoidal}

The current gold standard for recurrent variceal haemorrhage after failed medical treatment and local endoscopic banding or sclerotherapy is the transjugular intrahepatic stent shunt (TIPS), which has been widely used for over ten years, often with associated left gastric vein embolisation (Fig. 4). A long-term review of the management role for TIPS has been published, supporting its continued role after failed endoscopic and medical therapy. ${ }^{4}$ The tendency to occlude is one major drawback, although many can be salvaged by clearing thrombus and re-dilation (Fig. 5)

Another report analyses the efficiency in preventing re-bleeding (recurrent variceal bleeding $27 \%$ at 5 years) and patency rates (a poor primary patency of $22 \%$ at one year; primary assisted patency $46 \%$ at 5

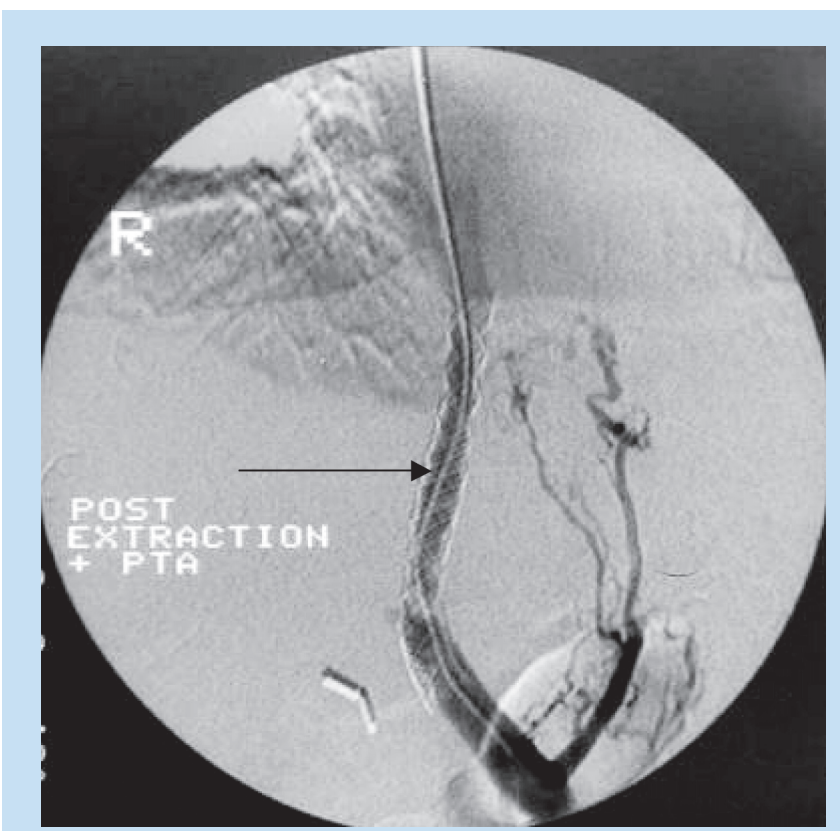

Fig. 5b. Re-opened TIPS (arrow) following aspiration thrombectomy and angioplasty.

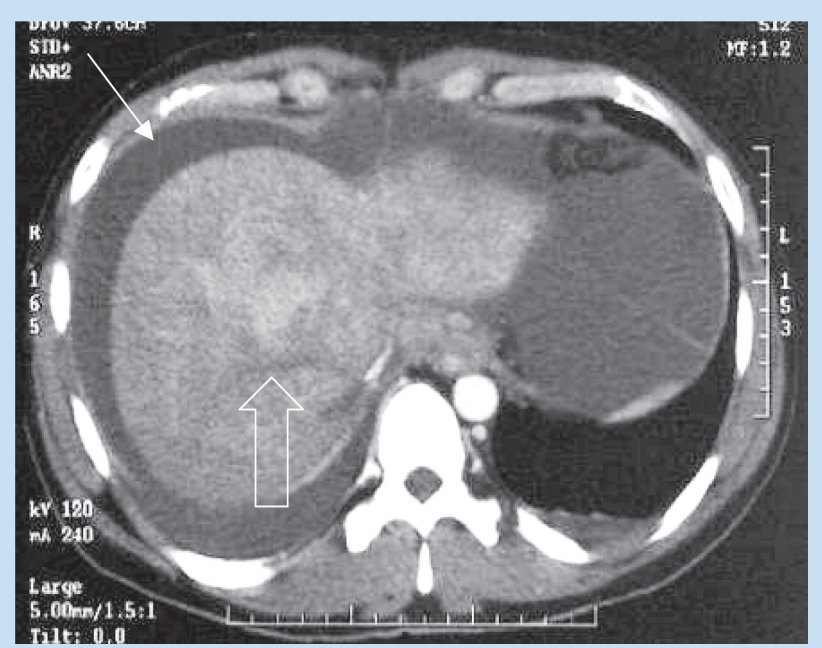

Fig. 6a. Acute Budd-Chiari with ascites (arrow), patchy liver perfusion and occluded right hepatic vein (open arrow).

years, and secondary $91 \%$ at 5 years). Encephalopathy rates were $20 \%$ for chronic or severe intermittent encephalopathy, and $52 \%$ overall, both at 3 years. Even more sobering is the overall patient survival of $42 \%$ at 5 years. 5

The problem of incapacitating encephalopathy has led to a variety of flow-reducing techniques, including hour-glass chokes in the preexisting stents. ${ }^{6}$

\section{Post sinusoidal}

For the acute Budd-Chiari syndrome (Fig. 6), thrombolysis for fresh thrombosis (again, either given arterially, via the superior mesenteric artery, or directly into the thrombus in the hepatic veins), and mechanical or aspiration thrombectomy are sometimes feasible. ${ }^{7}$ 


\section{PICTORIAL REVIEW}

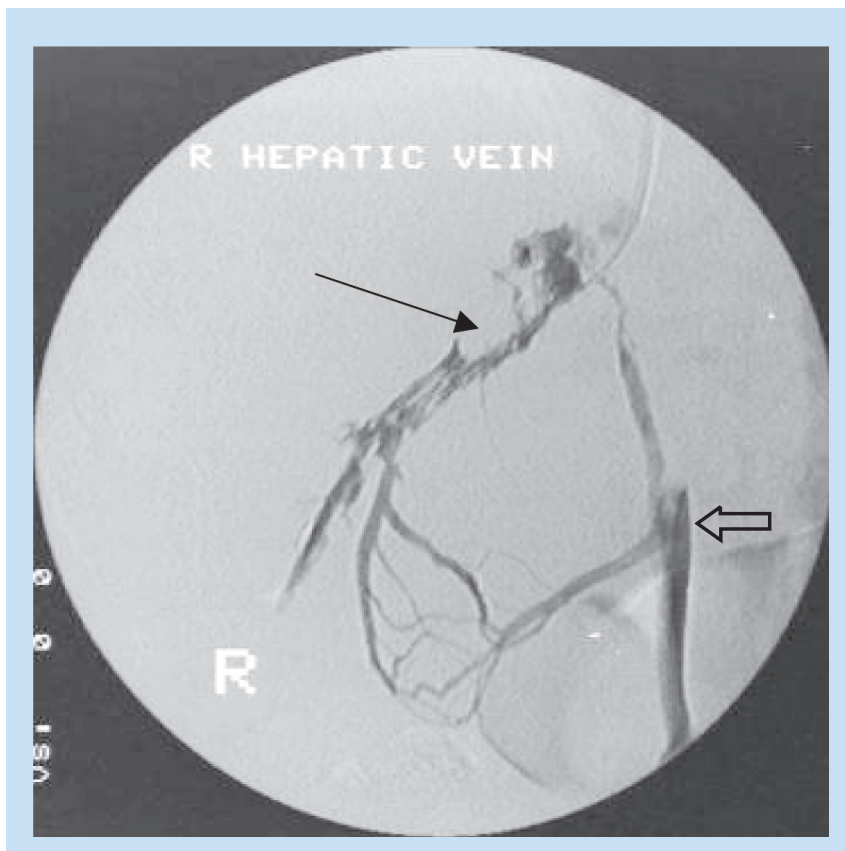

Fig. 6b. Clot in right hepatic vein (arrow) in a different patient with acute Budd-Chiari syndrome. Note filling of middle hepatic vein (open arrow) as a collateral drainage route.

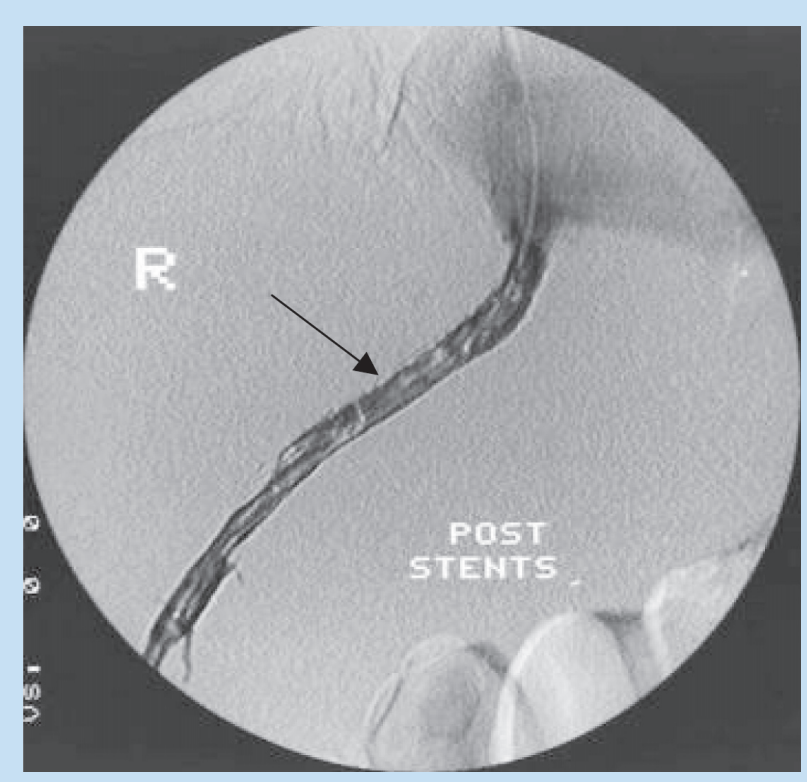

Fig. 6c. Recanalised right hepatic vein using stents (arrow).

Angioplasty or stenting of local hepatic vein or inferior vena cava (IVC) stenoses is seldom possible, as suitable lesions are infrequent. The ideal web-like narrowing can, however be opened up and kept patent for a few years by angioplasty and stenting (Fig. 7).

TIPS has also been used in both the acute and chronic (Fig. 8) forms of the Budd-Chiari syndrome, with the proposed benefit arising by providing a route for venous decompression. ${ }^{7,8}$ With absent hepatic veins, a more risky puncture from the IVC to the portal vein may be required (Fig. 9); there may also be associated portal vein thrombosis in these patients.

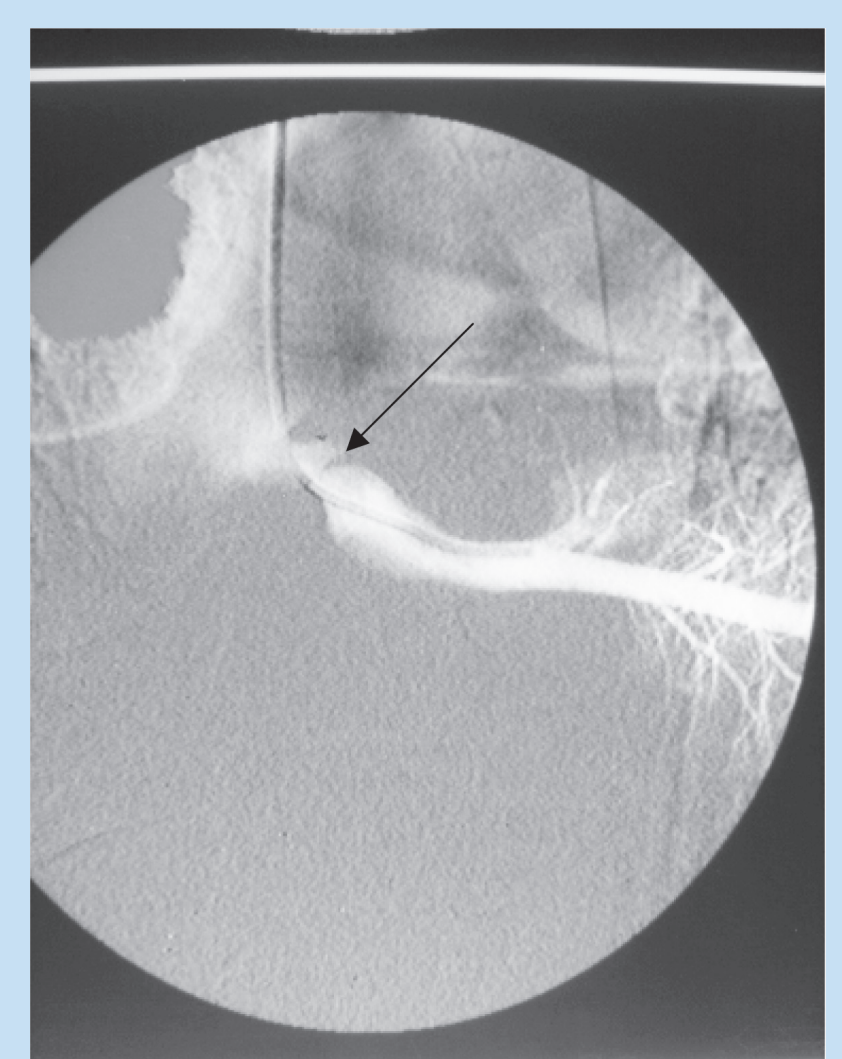

Fig. 7a. Left hepatic venogram showing central web-like stenosis (arrow).

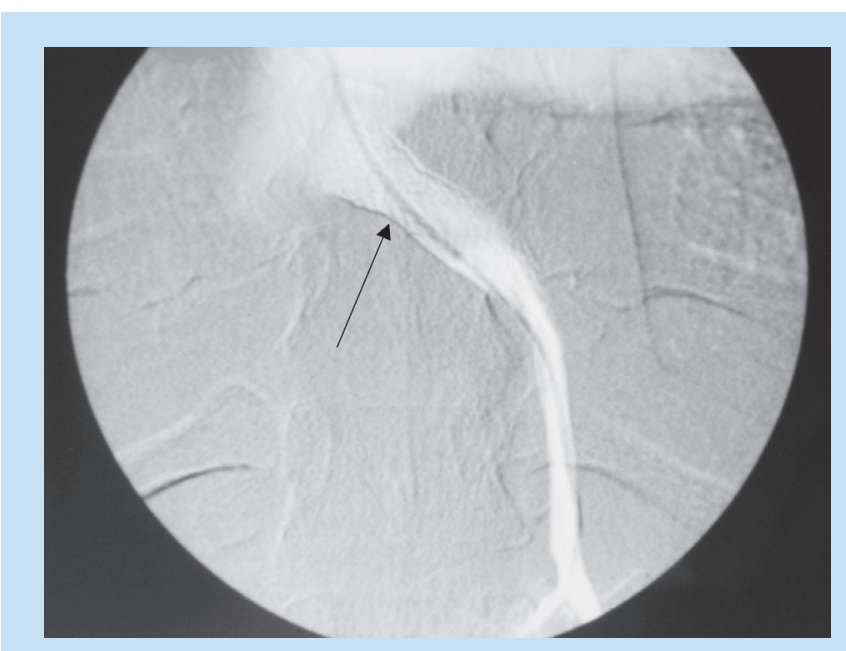

Fig. 7b. After successful treatment by metal stent (arrow). Note that another branch of the left hepatic vein has been catheterised.

\section{Newer ideas}

Covered stents for TIPS appear to offer substantially better patencies of over $80 \%$ at one year. ${ }^{9,10}$ The belief is that the covered portion of the stent prevents bile contact with the TIPS track, theoretically limiting shunt occlusion by thrombosis or endothelial hyperplasia.

TIPS placement has been successful in patients with recent portal vein thrombosis (Fig. 10). ${ }^{11}$ Placement of TIPS in patients with cavernous 


\section{PICTORIAL REVIEW}

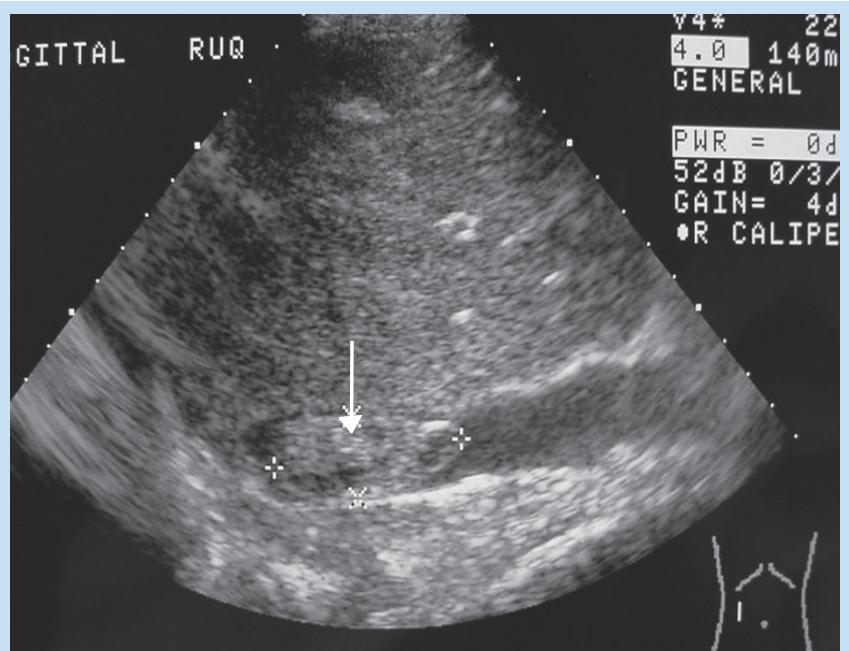

Fig. 8a. Chronic Budd Chiari with large chronic IVC filling defect (arrow) seen (a) on ultrasound, and

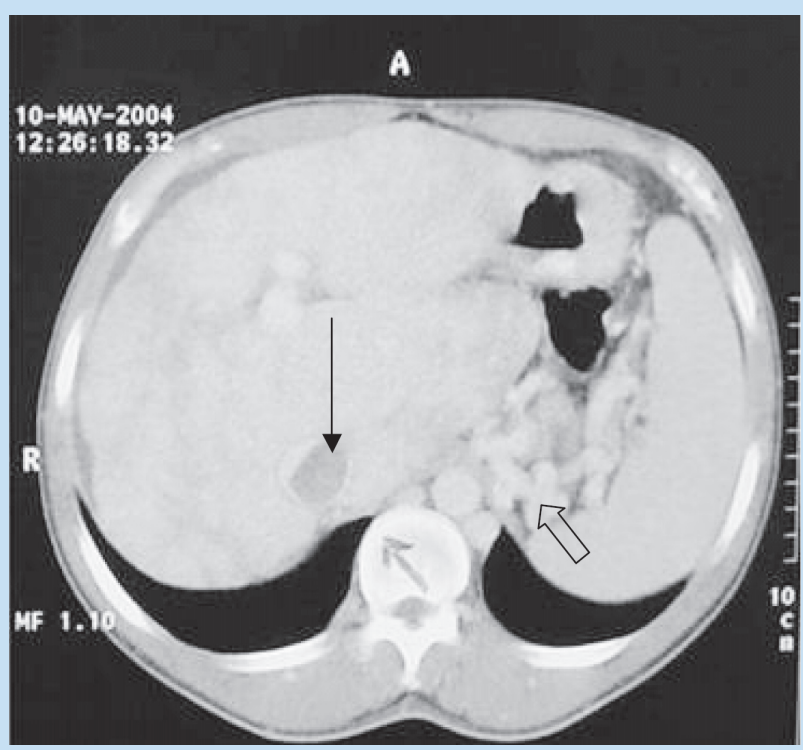

(b) on CT. Note large portosystemic collaterals (open arrow) on the CT

transformation of the portal vein, with resultant portal biliopathy has recently been described. ${ }^{12}$ Portal biliopathy (Fig. 11) arises as a consequence of the compressive effect of the cavernous transformation on the biliary tree, normally requiring a surgical shunt. ${ }^{13}$

Percutaneous Retroperitoneal Splenorenal Shunt (PRESS), involving percutaneous recreation of a reversed splenorenal (Warren) type shunt, although described 10 years ago in animals, ${ }^{14}$ has not yet had any clinical impact. A newer technique of direct portosystemic shunt creation has recently been performed in pigs, ${ }^{15}$ but has likewise not yet been adopted in day-to-day practice.

Gastric varices have been treated by a novel approach using the left renal and adrenal veins for access to the gastrorenal collaterals, and employing balloon catheters and cyanocrylate to occlude these veins. ${ }^{16}$ Azygous vein access could potentially allow similar access to the gastrooesophageal veins via a transjugular approach.

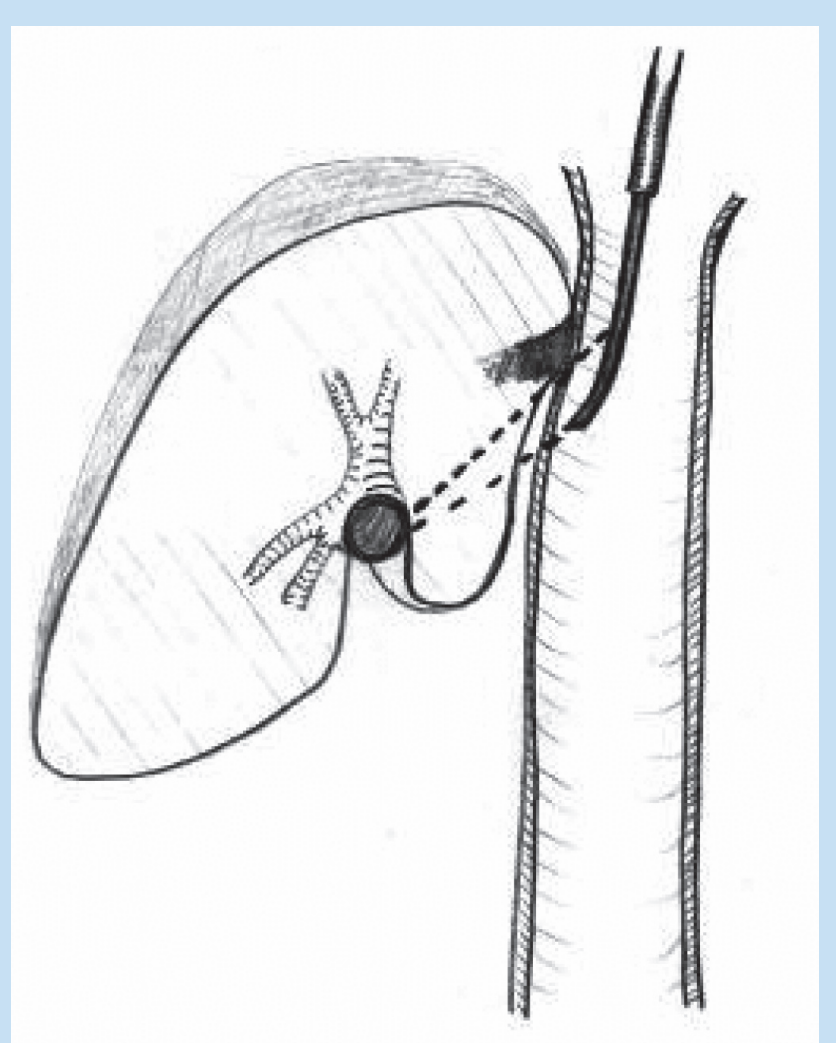

Fig. 9. Potential TIPS placement from the IVC

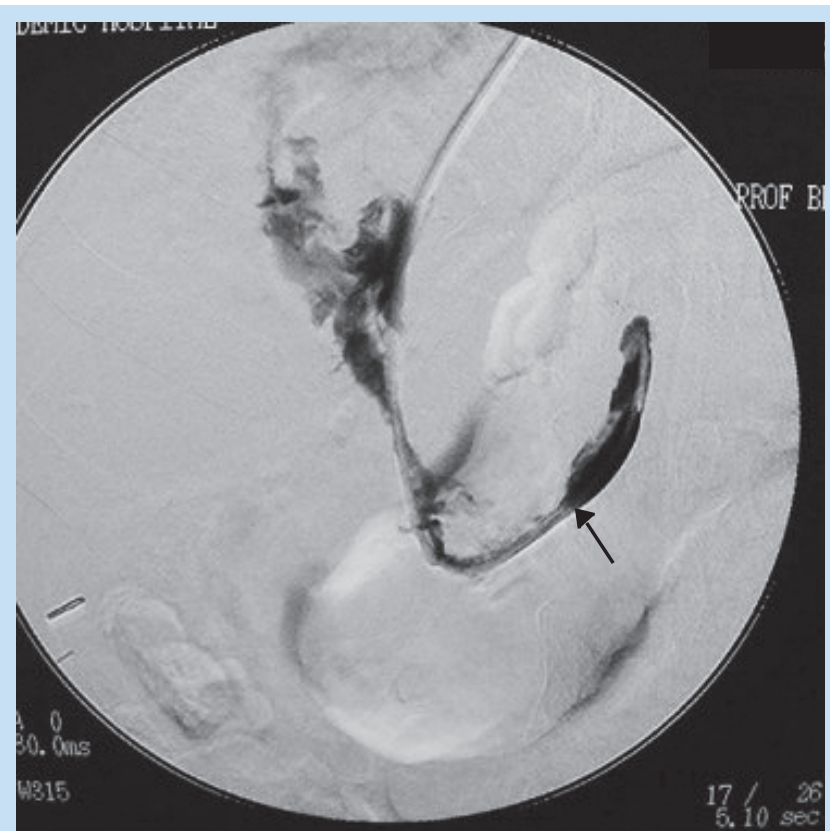

Fig. 10. Thrombosed portal and splenic vein (arrow) discovered during attempted TIPS placment.

The previous transhepatic approach to varices has largely been abandoned because of complications, but may re-surface with image guidance and micropuncture sets reducing the risks. Left gastric venous 


\section{PICTORIAL REVIEW}

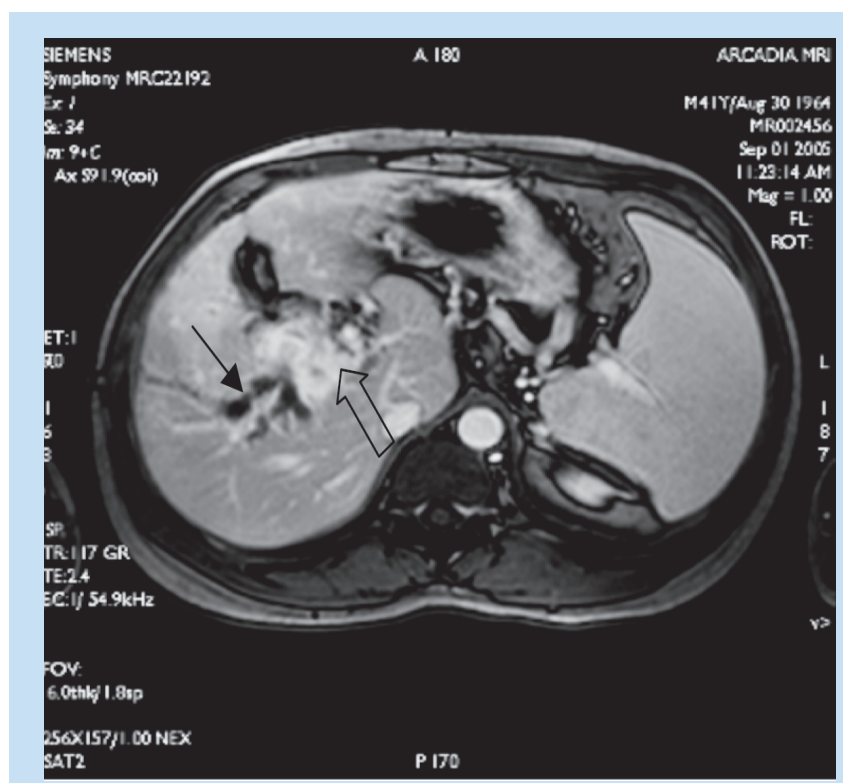

Fig. 11a. Portal biliopathy - note the cavernous transformation (open arrow) and dilated intrahepatic ducts (arrow).

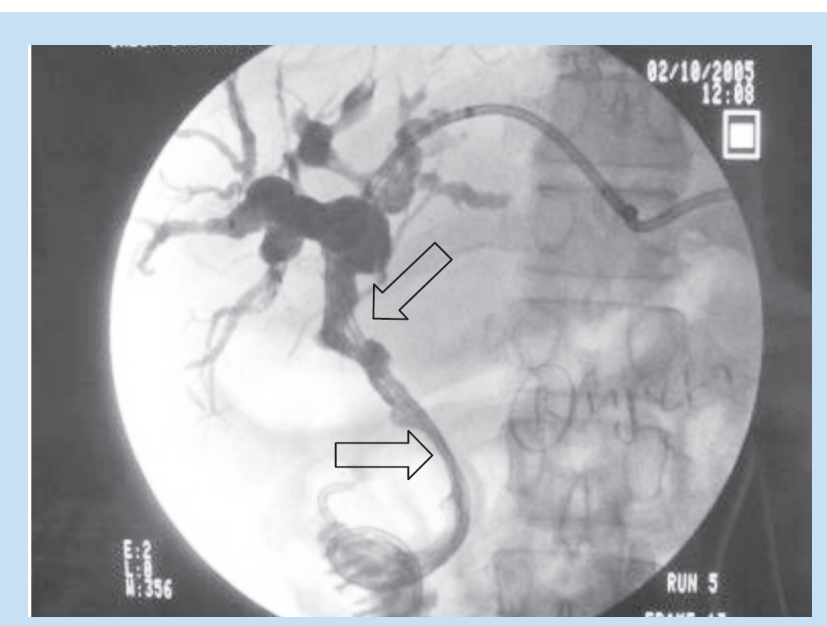

Fig. 11b. Cholangiogram showing long segment extrahepatic narrowings (open arrows) due to cavernous transformation in portal biliopathy.

embolisation is probably worth performing after all TIPS procedures, to avoid having to perform a separate procedure should re-bleeding occur.

\section{Conclusion}

The various options available are seldom widely applicable, apart from the TIPS procedure. Increasing experience has shown the limited longterm efficiency of TIPS without re-intervention, but covered stents appear to offer better patency rates. Encephalopathy remains a concern. Newer options allow expansion into treating gastric varices, as well as procedures recreating radiological shunts analogous to surgical shunts.

Radiological intervention expands the options available to some patients with portal hypertension, but increased awareness of problems in the longer term has partially tempered enthusiasm in some areas, notably TIPS. However, newer developments may help address some of these issues.

1. Guglielmi A, Fior F, Halmos $\mathrm{O}$, et al. Transhepatic fibrinolysis of mesenteric and portal vein thrombosis in a patient with ulcerative colitis: a case report. World J Gastroenterol 2005; 11:2035-2038.

2. Ueda $\mathrm{M}$, Egawa $\mathrm{H}$, Ogawa $\mathrm{K}$, et al. Portal vein complications in the long-term course after pediatric living donor liver transplantation. Transplant Proc 2005; 37:1138-1140.

3. Madoff DC, Denys A, Wallace MJ, et al. Splenic arterial interventions: anatomy, indications, technical considerations, and potential complications. Radiographics 2005; 25: Suppl 1, S191-S211.

4. Jalan R, Lui HF, Redhead DN, Hayes PC. TIPSS 10 years on. Gut 2000; 46:578-581.

5. ter Borg PC, Hollemans M, Van Buuren HR, et al. Transjugular intrahepatic portosystemic shunts: longterm patency and clinical results in a patient cohort observed for 3-9 years. Radiology 2004; 231:537-545.

6. Madoff DC, Wallace MJ, Ahrar K, Saxon RR. TIPS-related hepatic encephalopathy: management options with novel endovascular techniques. Radiographics 2004; 24(1):21-36.

7. Griffith JF, Mahmoud AE, Cooper S, Elias E, West RJ, Olliff SP. Radiological intervention in Budd-Chiari syndrome: techniques and outcome in 18 patients. Clin Radiol 1996; 51:775-784.

8. Ganger DR, Klapman JB, McDonald V, et al. Transjugular intrahepatic portosystemic shunt (TIPS) for Budd-Chiari syndrome or portal vein thrombosis: review of indications and problems. AmJ Gastroenterol 1999; 94:603-608.

9. Gandini R, Konda D, Simonetti G. Transjugular intrahepatic portosystemic shunt patency and clinical outcome in patients with Budd-Chiari syndrome: covered versus uncovered stents. Radiology 2006; 241:298-305.

10. Rossle M, Siegerstetter V, Euringer W, et al. The use of a polytetrafluoroethylene-covered stent graft for transjugular intrahepatic portosystemic shunt (TIPS): Long-term follow-up of 100 patients. Acta Radiol 2006; 47:660-666.

11. Radosevich PM, Ring EJ, LaBerge JM, et al. Transjugular intrahepatic portosystemic shunts in patients with portal vein occlusion. Radiology 1993; 186:523-527.

12. Walser EM, Soloway R, Raza SA, Gill A. Transjugular portosystemic shunt in chronic portal vein occlusion: importance of segmental portal hypertension in cavernous transformation of the portal vein. $J$ Vasc Interv Radiol 2006; 17:373-378.

13. Chandra R, Kapoor D, Tharakan A, Chaudhary A, Sarin SK. Portal biliopathy. J Gastroenterol Hepatol 2001; 16:1086-1092.

14. Kaminou T, Rosch J, Yamada R, et al. Percutaneous retroperitoneal splenorenal shunt: an experimental study in swine. Radiology 1998; 206:799-802.

15. Arepally A, Karmarkar PV, Qian D, Barnett B, Atalar E. Evaluation of MR/fluoroscopy-guided portosystemic shunt creation in a swine model. J Vasc Interv Radiol 2006; 17:1165-1173.

16. Hirota S, Matsumoto S, Tomita M, Sako M, Kono M. Retrograde transvenous obliteration of gastric varices. Radiology 1999; 211:349-356. 